\title{
ENRAIZAMENTO DE ESTACAS DE LICHIA (Litchi chinensis SONN.)
}

\author{
S. LEONEL, J. D. RODRIGUES', S. D. RODRIGUES ${ }^{2}$ \\ ${ }_{2}^{1}$ Pós-Graduanda, FCACNNESP, Botucatu, SP. \\ ${ }^{2}$ Depto. de Botánica - I.B/UNESP - CEP: 18618-000 - Botucatu , SP
}

\begin{abstract}
RESUMO: Estudou-se os efeltos de auxinas exógenas e ácido borico, no enraizamento de estacas de lichla (Litchi chinensis Sonn.). As estacas foram uniformizadas, com $25 \mathrm{~cm}$ de comprimento e 4 folhas cortadas pela metade. Cerca de 2,5 cm da base das mesmas fol mergulhado nos tratamentos: $\mathrm{H}_{2} \mathrm{O}$; Boro $150 \mu \mathrm{g} / \mathrm{ml}$;BA 5.000 ppm, IBA 2.000 ppm; IBA 5.000 ppm + Bon $150 \mu \mathrm{g} / \mathrm{ml}$;BA 2.000 ppm + Boro $150 \mu \mathrm{g} / \mathrm{ml}$; NAA 3.000 ppm; NAA 1.500 ppm; NAA 3.000 ppm + Boro $150 \mu \mathrm{g} / \mathrm{ml}$; NAA $1.500 \mathrm{ppm}$ + Boro $150 \mu \mathrm{q} / \mathrm{ml}$. A estaquia foi realizada no mês de setembro (Hemisfério sul), sendo que as estacas foram colocadas em handejas de isopor, tendo como substrato vermiculita e mantidas sob nehulizaçăo intermitente. Os resultados obtides permitiram concluir que o IBA 5.000 ppm por 1 minuto foi o tratamento mais efetivo, proporcionando 83,33\% de estacas enraizadas em 120 dias, enquanto o tratamento testemunha $\left(\mathrm{H}_{2} \mathrm{O}\right.$ ), apresentou somente $16,67 \%$ de estacas enraizadas.
\end{abstract}

Descritores: reguladores vegetais, ácido bórico, "callus", lichieira, estacas; enraizamento

\section{ROOTING OF LYCHEE (Litchi chinensis SONN.) CUTTINGS}

\begin{abstract}
The effects of exogen auxins and boric acid were studied on lychee (Litchi chinensis Sonn.) cuttings. Cuttings were standardized to twenty-five $\mathrm{cm}$ length, with four leaves, cut in half. The bases of the cuttings were dipped of $2,5 \mathrm{~cm}$ in water solutions, resulting in the following treatments: $\mathrm{H}_{2} \mathrm{O}$; Boron $150 \mu \mathrm{g} / \mathrm{ml}$;BA $5,000 \mathrm{ppm}$;BA 2,000 ppm; IBA $5,000 \mathrm{ppm}$ plus boron $150 \mu \mathrm{g} / \mathrm{ml}$; IBA 2,000 ppm plus boron $150 \mu \mathrm{g} / \mathrm{ml}$; NAA 3,000 ppm; NAA 1,500 ppm; NAA 3,000 ppm plus boron $150 \mu \mathrm{g} / \mathrm{ml}$; NAA 1,500 ppm plus boron $150 \mu \mathrm{g} / \mathrm{ml}$. Cuttling was performed in September (southern hemisphere) and the cuttings were place in styrofoam trays, using vermiculite as substratum and kept under intermittent mist. It was concluded that 5,000 ppm IBA for one minute was the best treatment to improve rooting (83,33\%), while the control $\left(\mathrm{H}_{2} \mathrm{O}\right)$ showed only $16,67 \%$ of rooted cuttings.
\end{abstract}

Key Words: growth regulators, boric acid, "callus", lychee, cuttings

\section{INTRODUÇĀO}

A lichia é uma fruta exótica, de origem chinesa, que está tornando-se bastante conhecida no Brasil, onde vem sendo muito procurada. Contudo, o que se observa é a existência de poucos produtores dessa frutifera em nosso pais, devido principalmente dis dificuldades de propagação.

A propagação através de sementes retarda a produção, devido ao longo periodo improdutivo ocasionado pela juvenilidade, tendo também a desvantagem de que as sementes dessa espécie perdem rapidamente o poder germinativo (BAILEY, 1927; COBIN, 1954; GOMES, 1987; HARTMANN \& KESTER, 1983; SINGH et al., 1963). Além disso, muitas plantas originadas de sementes têm pequena produção, com frutas de baixa qualidade (YEE, 1957), sendo que tais fatores desfavorecem um maior interesse por parte dos fruticultores.
O enraizamento de estacas é uma das alternativas para a propagação dessa espécie, uma vez que permite o início da produção de fruta num menor espaço de tempo, além de permitir a manutenção das características desejáveis selecionadas nas matrizes, embora em alguns casos, seja um processo dificil e demorado (MENZEL, 1985). Para acelerar e promover o enraizamento de estacas, habitualmente são empregados hormônios do grupo das auxinas, os quais levam à uma maior porcentagem de formação de raizes, melhor qualidade das mesmas e uniformidade no enraizamento (HARTMANN \& KESTER, 1983).

O presente trabalho, foi conduzido com o objetivo de avaliar o efeito da utilização de fitorreguladores, como o ácido indol-butírico e alfanaftaleno-acético, aplicados isoladamente ou $\mathrm{em}$ conjunto com o ácido bórico, o qual de acordo com diversos autores (HIRSCH et al., 1982; JARVIS et al., 
1983; LEWIS, 1980; MIDDLETON, 1977), exerceria um efeito sinergístico com as auxinas, no desenvolvimento de raizes em estacas de lichieira (Litchi chinensis Sonn.).

\section{MATERIAL E MÉTODOS}

O experimento foi realizado em câmara de nebulização, sob ripado, do Departamento de Horticultura, da Faculdade de Ciências Agronômicas, do Campus de Botucatu, UNESP - SP.

As estacas foram retiradas de ramos terminais de lichieira com 18 anos de idade, pertencentes ao pomar da Fazenda Experimental Lageado e padronizadas de modo a apresentarem um comprimento de $25 \mathrm{~cm}$ (RAM \& MAJUMDAR, 1983), sendo mantidas 4 folhas cortadas pela metade.

A época para retirada das estacas das plantas matrizes correspondeu ao mês de setembro de 1988. Foram utilizadas 360 estacas de lichieira, devidamente identificadas e distribuidas aleatoriamente, sendo cada tratamento constituído por 9 estacas por parcela, com 4 repetições, num delineamento inteiramente casualizado.

Os tratamentos utilizados, foram os seguintes:

. Tl $\left(\mathrm{H}_{2} \mathrm{O}\right)-03$ horas

. T2 (Boro $150 \mu \mathrm{g} / \mathrm{ml}$ ) - 03 horas

. T3 (IBA $5.000 \mathrm{ppm}$ ) - 01 minuto

. T4 (IBA $2.000 \mathrm{ppm}$ ) - 05 minutos

. T5 (IBA $5.000 \mathrm{ppm}+$ Boro $150 \mu \mathrm{g} / \mathrm{ml}$ ) - 01 hora

. T6 (IBA $2.000 \mathrm{ppm}+$ Boro $150 \mu \mathrm{g} / \mathrm{ml}$ ) - 01 hora

. T7 (NAA 3.000 ppm) - 01 minuto

. T8 (NAA $1.500 \mathrm{ppm}$ ) - 05 minutos

. T9 (NAA $3.000 \mathrm{ppm}+$ Boro $150 \mu \mathrm{g} / \mathrm{ml}) \cdot 30$ minutos

. T10 (NAA $1.500 \mathrm{ppm}+$ Boro $150 \mu \mathrm{g} / \mathrm{ml}) \cdot 30$ minutos

O ácido alfa-naftaleno-acético foi utilizado na forma do produto comercial Nafusaku, contendo $20 \%$ de NAA.

O tratamento constou da imersão de 2,5 $\mathrm{cm}$ da base das estacas em soluções preparadas com os fitorreguladores e o ácido bórico, sendo que nos tratamentos com o NAA e com o boro utilizou-se água destilada para a diluição, e nos tratamentos com - IBA utilizou-se uma mistura de 1:1 de álcool etílico $99 \%$ e água.

A seguir, as estacas foram colocadas para enraizar $\mathrm{em}$ bandejas de isopor de $12 \mathrm{~cm}$ de profun- didade, tendo como substrato vermiculita de granulação média e mantidas sob nebulização.

Os parâmetros avaliados foram: porcentagem de sobrevivência das estacas, porcentagem de formação de calos, porcentagem de enraizamento $e$ comprimento médio das raizes $(\mathrm{mm})$, sendo as avaliações realizadas aos 120 dias após a instalação do experimento.

\section{RESULTADOS E DISCUSSĀO}

O emprego de auxinas exógenas, visando favorecer ou acelerar o enraizamento de estacas, já foi comprovado em várias frutiferas, havendo entretanto, poucos trabalhos no que se refere à estaquia da lichieira.

Os resultados obtidos com este trabalho, mostram um incremento na porcentagem de estacas enraizadas (Figura 1), através do uso do ácido indolbutírico $5.000 \mathrm{ppm} / 01$ minuto, o que vem concordar com os resultados de BHANDARY \& SHIVASHANKAR (1970), que conseguiram alta porcentagem de enraizamento, utilizando estacas de lichieiras tratadas com IBA $5.000 \mathrm{ppm}$, antes de serem colocadas sob névoa. Já Lenka \& Das (1981), citados por MENZEL (1985) utilizaram IBA 3.000, 6.000 e $9.000 \mathrm{ppm}$, obtendo $32,8 \%$ de estacas enraizadas com IBA $3.000 \mathrm{ppm}$, vindo comprovar a citação do mesmo autor, sobre a dificuldade de enraizamento dessa espécie.

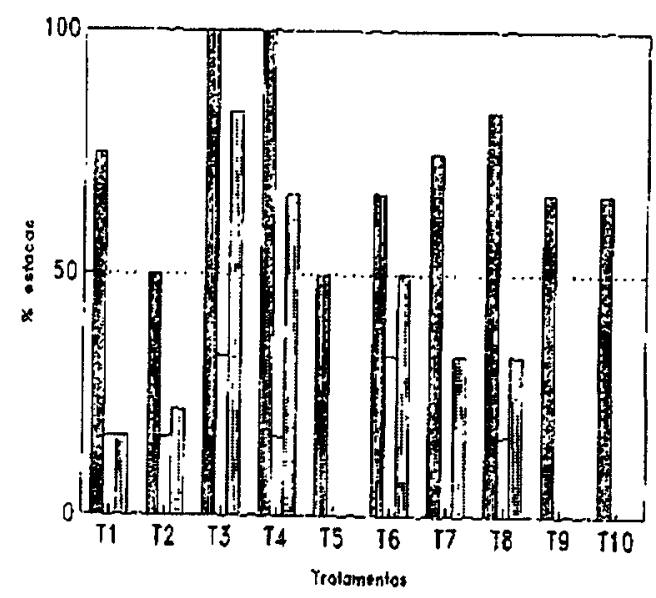

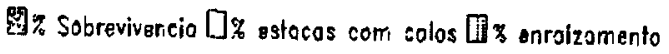

Figura 1. Resultados obtidos para \% de sobrevivência, de estacas com calos e de enraizamento de estacas de lichia, aos 120 dias. 
Comprimento (mm)

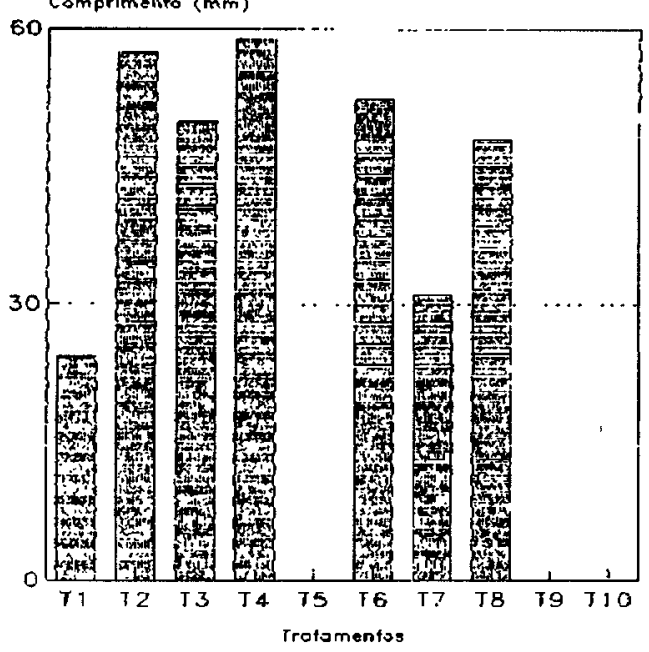

Figura 2 Resultados obtidos para comprimento das raizes formadas em estacas de lichieira, aos 120 dias

O sucesso no enraizamento das estacas depende de inúmeros fatores, incluindo época de estaquia, temperatura, concentração dos fitorrcguladores, tempo de imersão das estacas nos tratamentos, etc., sendo que a escolha de uma boa combinação do binómio concentração/tempo de imersão, não é uma tarefa fácil, havendo inclusive, controvérsias na literatura, com relação a algumas espécies.

IRITANI (1981), sugere que estacas herbáceas devem ser tratadas com baixas concentrações de auxinas, enquanto que para estacas lenhosas e de dıficil enraizamento, como é o caso das estacas de lichieira, a concentração deve ser alta, próxima à fitotóxica. Contudo, neste trabalho, pode observarse o efeito desfavorável do emprego de altas concentrações (IBA $5.000 \mathrm{ppm}+$ Boro/60 minutos; NAA $1.500 \mathrm{ppm}+$ Boro/30 minutos), em tempos de imersão prolongados. $\mathrm{O}$ uso desses fitorreguladores em altas concentrações num tempo de imersão também alto, teve efeitos contrários ao enraizamento, não devendo portanto, ser recomendado.

Nesses tratamentos, observou-se inicialmente uma intensa queda de folhas, seguida de descorticamento da base das estacas. Diferentes pesquisadores, concordam que para um bom enraizamento é necessária a presença de folhas e ou gemas nas estacas (WEAVER, 1982); portanto, podese também, atribuir o fato do não enraizamento das estacas as quais receberam esses tratamentos, à falta de folhas. Trabalhando com estacas de pesseguei- ro, FACHINELLO \& KERSTEN (1981), observaram que a aplicação exógena de auxinas não teve efeito naquelas que não possuiam folhas.

A utilização do ácido bórico, aplicado isoladamente, não se mostrou ser tão eficiente na indução do enraizamento $(22,22 \%)$ e nem na sobrevivência das estacas $(50,00 \%)$, vindo a corroborar a literatura existente. Para MURRAY et al. (1957), o boro não tem efeito na emissão de raízes, não tendo com isso, efeito similar ao dos reguladores vegetais, sendo essencial para o desenvolvimento das raízes. Tal fato, pode ser verificado através das Figuras $1 \mathrm{e}$ 2 , nas quais esse elemento apesar de não aumentar o enraizamento, promoveu o crescimento de raizes com o segundo maior comprimento médio das raízes (57,50 mm), em detrimento somente do obtido com a utilização do IBA $2.000 \mathrm{ppm}(59,00 \mathrm{~mm})$.

O tempo de imersão se mostrou ser adequado para o IBA $5.000 \mathrm{ppm}$ e para o IBA 2.000 $\mathrm{ppm}$, dados esses refletidos na máxima porcentagem de sobrevivência $(100 \%)$ e no melhor enraizamento das estacas ( $83,33 \%$ e $66,67 \%$, respectivamente).

No que se refere à formação de calos, o que se observou foi uma baixa porcentagem de estacas com calos em praticamente todos os tratamentos (Figura 1), podendo tais resultados serem atribuídos à dificuldade da espécie para emissão dos calos ou então, adicionado a isso, ao tempo de imersão considerado fitotóxico em alguns tratamentos. Entretanto, é importante lembrar que, de acordo com SILVA (1985), a rapidez na formação de calo, nem sempre está relacionado com a formação de raízes, uma vez que são processos fisiológicos independentes. HARTMANN \& KESTER (1983), relatam que em lenho perene, onde já estão presentes xilema e floema secundários, as raizes adventícias têm origem, geralmente, do tecido jovem do floema secundário, mas também podem originar-se os raios vasculares, câmbio, ou dos calos produzidos na base das estacas. $\grave{A}$ semelhança do ocorrido com a porcentagem de estacas enraizadas, porém em proporções menores, o tratamento com IBA $5.000 \mathrm{ppm} / 01$ minuto, proporcionou a maior porcentagem de estacas com calos $(33,33 \%)$, todavia igualando-se, neste parâmetro, ao IBA $2.000 \mathrm{ppm}+$ Boro/01 hora $(33,33 \%)$.

\section{CONCLUSÕES}

Através dos resultados obtidos, foi possível concluir que:

- O emprego do IBA (ácido indol-butírico) foi superior ao do NAA (ácido alfa-naftaleno-acético), sen- 
do a maior porcentagem de estacas enraizadas $(83,33 \%)$, obtida com IBA 5.000 ppm por 1 minuto;

- O mês de setembro foi uma época favorável para a sobrevivência e para o enraizamento das estacas;

- O tempo de imersão das estacas nas soluções foi supra-ótimo para o $\mathbb{B B A} 5.000 \mathrm{ppm}+$ Boro $150 \mu \mathrm{g} /$ $\mathrm{ml}$ por 60 minutos; para o NAA $3.000 \mathrm{ppm}+$ Boro $150 \mu \mathrm{g} / \mathrm{ml}$ por 30 minutos e para o NAA $1.500 \mathrm{ppm}$ + Boro $150 \mu \mathrm{g} / \mathrm{ml}$ por 30 minutos.

\section{REFERÊNCLAS BIBLIOGRÁFICAS}

BAILEY, L.H. In: STANDARD Cyclopedia of Horticulture. London: Macmillan, 1927. p.3639.

BHANDARY, K.R.; SHIVASHANKAR, T.T. Propagation of litchi undermist. South Indian Horticulture, v.18, p. 74-76, 1970.

COBN, M. The lytchee in Florida. University of Florida. Agricultural Experiment Stations. Gainesville. Bulletin, 471, 1954. 35p.

FACHINELLO, J.C.; KERSTEN, E. Efeito do ácido indolbutírico na porcentagem de estacas semi-lenhosas enraizadas de pessegueiro (Prunus persica (L.) Batsch.), cv Diamante, em condiçð̃es de nebulização. Revista Brasileira de Fruticultura, Cruz das Almas, v.3, p.49-50, 1981.

GOMES, P. Fruticultura brasileira. 2.ed. São Paulo: Nobel, 1987. 448p.

HARTMANN, H.T.; KESTER, D.E. Plant propagation: principle and practices. New York: Englewood Clipps; Prentice-Hall, 1983. 727p.

HIRSCH, A.M.; PENGELLY, W.L.; TORREY, J.G. Endogenous IAA levels in boron-deficient and control root tips of sunflower. Botanical Gazette, Chicago, v.143, p.15-19, 1982
IRITANI, G. Ação de reguladores de crescimento na propagação por estaquia de Ilex paraguariensis Saint Hilaire e Araucaria angustifolia (Bert.) O. Ktze. Curitiba, 1981. 163p. Dissertação (Mestrado) - Universidade Federal do Paraná.

JARVIS, B.C.; ALI, A.H.N.; SHAHEED, A.I. Auxin and boron in relation to the response and ageing of mung bean cuttings. New Phytologist, London, v.95, p.509-518, 1983.

LEWIS, D.H. Boron, lignification and the origin of vascular plants:- a unified hypothesis. New Phytologist, London, v.84, p.209-229, 1980.

MENZEL, C.M. Propagation of lychee: a review. Scientia Horticulturae, Amsterdam, v.25, p.31-48, 1985.

MIDDLETON, W. Root development in cuttings of Phaseolus aureus Roxb. Sheffield, 1977. 167p. Thesis (Ph.D.) - University of Sheffield.

MURRAY, H.R.; TAPER, C.D.; PICKUP, T.; NUSSEY, A.N. Boron nutrition of softwood cuttings of geranium and currant relation to root development. Proceeding American Society for Horticultural Science, Geneva, v.69, p.498-501, 1957.

RAM, M.; MAJUMDAR, D.K. Effect of indole butyric acid on stooling in lychee. Indian Journal of Horticultural, Bangalore, v.40, n.314, p.211-212, 1983.

SILVA, I.C. Propagação vegetativa: aspectos morfo-fisiológicos. Boletim técnico CEPLAC, Itabuna, v.4, p.1-26, 1985.

SINGH, S.; KRISHNAMURTH, S.; KATYAL, S.L. Fruticulture in India. New Delhi: ICAR, 1963. 445p.

WEAVER, R.Y. Reguladores del crecimiento de las plantas en la agricultura. 2.ed. Barcelona: Trillas, 1982. 540p.

YEE, W. The lychee in Hawaii. Extension Circular Hawail Agricultural Experiment Station., Honolulu, n.366, p.1-13, 1957.

Recebido para publicação em 09.01.95

Aceito para publicação em 14.07.95 